\title{
High power diode laser surface glazing of concrete.
}

\author{
J. Lawrence and L. Li \\ Manufacturing Division, Department of Mechanical Engineering, University of Manchester \\ Institute of Science and Technology (UMIST), Manchester, M60 1QD, UK.
}

\section{Correspondence}

Dr. Jonathan Lawrence

Manufacturing Division,

Department of Mechanical Engineering,

University of Manchester Institute of Science and Technology (UMIST),

Manchester,

M60-1QD,

UK.

Tel : (+44) $0161236-3311$ ext. 2383

Fax : (+44) 0161 200-3803

e-mail : j.lawrence@stud.umist.ac.uk 


\section{ABSTRACT}

This present work describes the utilisation of the relatively novel high power diode laser (HPDL) to generate a surface glaze on the ordinary Portland cement (OPC) surface of concrete. The value of such an investigation would be to facilitate the hitherto impossible task of generating a durable and long-lasting surface seal on the concrete, thereby extending the life and applications base of the concrete. The basic process phenomena are investigated and the laser effects in terms of glaze morphology, composition and microstructure are presented. Also, the resultant heat affects are analysed and described, as well as the effects of the shield gases, $\mathrm{O}_{2}$ and Ar, during laser processing. HPDL glazing of OPC was successfully demonstrated with power densities as low as $750 \mathrm{~W} \mathrm{~cm}^{-2}$ and at scanning rates up to $480 \mathrm{~mm} \mathrm{~min}^{-1}$. The work showed that the generation of the surface glaze resulted in improved mechanical and chemical properties over the untreated OPC surface of concrete. Both untreated and HPDL glazed OPC were tested for pull-off strength, rupture strength, water absorption, wear resistance and corrosion resistance. The OPC laser glaze exhibited clear improvements in wear, water sorptivity, and resistance (up to $80 \%$ concentration) to nitric acid, sodium hydroxide and detergent. Life assessment testing revealed that the OPC laser glaze had an increase in actual wear life of 1.3 to 14.8 times over the untreated OPC surface of concrete, depending upon the corrosive environment.

Keywords: high power diode laser; HPDL; concrete; cement; glazing 


\section{INTRODUCTION}

Owing to their unique characteristics, lasers have the propensity to be employed for the noncontact processing of materials which are otherwise difficult to process. Concrete is a composite material consisting of an array of fine and coarse aggregate pieces embedded within an ordinary Portland cement (OPC) matrix. Consequently the processing and surface treatment of concrete can be an arduous task.

To date, many studies have been carried out to investigate the laser processing of concrete. Most of the research, however, has concentrated on the laser cutting of concrete and reinforced concrete using high power $\mathrm{CO}_{2}$ lasers, most prominently with regard to nuclear reactor decommissioning ${ }^{1-3}$. Also, as part of nuclear plant decommissioning, Li et al ${ }^{4-7}$ conducted research to determine the workability of several laser techniques for sealing/fixing radioactive contamination onto concrete surfaces. Such techniques experimented with were: direct glazing of the concrete, single and multiple layer fusion cladding and combined chemical/fusion cladding. Work by Sugimoto et $a l^{8}$ focused upon modifying the surface appearance and surface properties of cement based materials using a high power $\mathrm{CO}_{2}$ laser. The laser treatment produced novel surfaces, with surface textures, properties and appearance unique to laser treatment. The resultant physical characteristics and mechanical behaviour of the post-process cement based materials was later fully characterised by Wignarajah et al ${ }^{9}$. Borodina et al ${ }^{10}$ has carried out investigations into the structural changes within the composition of zirconia concrete caused by surface exposure to $\mathrm{CO}_{2}$ laser radiation, detailing microstructural changes, phase changes and the absorptivity characteristics. In all of these studies, spallation and excessive cracking and porosity formation were found to be major problems undermining the performance of the laser treated surface layer.

This present work is concerned with the utilisation of the relatively novel high power diode laser (HPDL) to generate a surface glaze on the OPC surface layer of concrete, and the effects thereof on the OPC's mechanical, physical and chemical properties. The ultimate aim of this investigation is to facilitate the hitherto impossible task of generating a durable and long-lasting surface seal on the concrete, thereby extending the life and applications base of 
the concrete. It is a distinct possibility that such a development may yield significant economic benefits.

\section{EXPERIMENTAL PROCEDURES}

The concrete studied in the experiments was the ubiquitous OPC based concrete. For the purpose of experimental convenience the as-received concrete blocks were sectioned into squares $(120 \times 120 \times 20 \mathrm{~mm})$ prior to laser treatment. The composition of the concrete by volume is as follows: $20 \mathrm{~mm}$ limestone aggregate (40\%), $10 \mathrm{~mm}$ limestone aggregate (14 \%), zone $\mathrm{M}$ sand (28.5\%), OPC (10.5\%) and particulate fine aggregate $(7 \%)$.

The laser used in the study was a surgical HPDL (Diomed Ltd.), emitting at $810 \mathrm{~nm} \pm 20 \mathrm{~nm}$ and operating in the $\mathrm{CW}$ mode with rated optical powers ranging from $0-60 \mathrm{~W}$. The laser beam was delivered to the work area by means of a $4 \mathrm{~m}$ long, $600 \mu \mathrm{m}$ core diameter optical fibre, the end of which was connected to a 2:1 focusing lens assembly mounted on the z-axis of a 3-axis CNC gantry table. The concrete sample blocks were irradiated using the defocused high order mode HPDL beam with a beam spot diameter of $2 \mathrm{~mm}$ and laser powers (measured at the workpiece using a Power Wizard power meter) of 20-55 W. The defocused laser beam was fired across the surfaces of the concrete samples by traversing the samples beneath the beam using the $\mathrm{x}$ - and $\mathrm{y}$-axis of the $\mathrm{CNC}$ gantry table at speeds ranging from $60-600 \mathrm{~mm} / \mathrm{min}$. The laser optics were protected by means of a coaxially blown shield gas jet a rate of $51 / \mathrm{min}$. For comparison purposes both $\mathrm{O}_{2}$ and Ar were used.

To determine the characteristics of the glazes the HPDL treated concrete samples were examined using optical microscopy, scanning electron microscopy (SEM), energy disperse $\mathrm{X}$-ray analysis (EDX) and x-ray diffraction (XRD) techniques, as well thermal analysis techniques. 


\section{GENERAL EFFECTS OF HIGH POWER DIODE LASER INTERACTION WITH CONCRETE}

\section{A. Effect of laser power density on melt depth and morphology}

Variations in the power density were seen to significantly affect the surface morphology and the size of the region of laser melting on the OPC surface of the concrete. A series of experiments were conducted with an $\mathrm{O}_{2}$ shield gas for a wide range of power densities, whilst the traverse speed was fixed at 120, 300 and $480 \mathrm{~mm} / \mathrm{min}$. Fig. 1 shows how the depth of the laser melt region on the OPC surface of the concrete increases with increased power density for the given traverse speeds.

In the relatively low power density range $(<0.75 \mathrm{~kW} / \mathrm{cm})$, a minimum power density level of around $0.2 \mathrm{~kW} / \mathrm{cm}$ was required, below which no discernible effect of the laser treatment on the OPC surface of the concrete could be seen, regardless of the traverse speed. A typical example of a low power density laser interaction region is the top track shown in Fig. 2. As one can see, laser interaction at this relatively low level resulted in only slight surface charring. At relatively medium power densities $(0.75-2.25 \mathrm{~kW} / \mathrm{cm})$, however, the surface condition of the OPC surface of the concrete after laser radiation had changed considerably. A typical example of a medium power density laser interaction region is the centre track shown in Fig. 2. Here a green coloured surface (which is discussed later) has been generated that appears glazed. The surface undulates greatly, with the undulations being regular in both periodicity and intensity. In addition, the surface displays very few microcracks and few porosities. The bottom track shown in Fig. 2 shows an example indicative of a relatively high power density range $(2.25-3 \mathrm{~kW} / \mathrm{cm})$ track. The effects in terms of surface condition are similar to those of the relatively medium power track. But, in contrast, the undulating surface is much more pronounced, whilst the surface is heavily pitted with many porosities and displays a large number of microcracks.

\section{B. Effect of traverse speed on melt depth and morphology}

As with power density variations, traverse speed changes affected both the surface morphology and the size of the region of laser interaction on the OPC surface of the concrete. 
A series of experiments were conducted with an $\mathrm{O}_{2}$ shield gas for a wide range of traverse speeds, whilst the power density was fixed at 1,2 and $3 \mathrm{~kW} / \mathrm{cm}$ with a spot diameter of $1 \mathrm{~mm}$. Fig. 3 shows the relationship between the depth of the laser treatment on the OPC surface of the concrete and the traverse speed for the stated power densities.

From the experiments it was observed that generally, as the traverse speed increased, the depth of the laser melt region decreased. Also, processing with traverse speeds less than $120 \mathrm{~mm} / \mathrm{min}$ and in excess of $480 \mathrm{~mm} / \mathrm{min}$, resulted in the surface condition of the OPC surface of the concrete after laser irradiation being extremely poor. As the tracks on the top and bottom of Fig. 4 show, the treated surfaces display many large porosities and a large number of microcracks.

\section{Mass loss/regain during and after laser treatment}

Although material ejection was generally not a typical feature of HPDL beam interaction with the OPC surface of the concrete, a loss in mass of the concrete was a possibility due to the resulting heat effects of the process. In order to determine any weight loss experienced by the concrete as a result of HPDL irradiation, a number of samples were stored in a controlled environment for two days prior to laser irradiation. The samples were weighed regularly to ensure a constant mass. The samples were treated at various power densities and traverse speeds and then immediately weighed. Fig. 5 shows the percentage loss of original mass experienced by the concrete in terms of power density and traverse speed.

As one can see from Fig. 5, the loss in mass experienced by the concrete increases almost proportionately in a linear manner with increasing power density up to approximately $2.1 \mathrm{~kW} / \mathrm{cm}$. After this point the loss in mass can be seen to decrease in terms of the power density. It is reasonable to assume that this indicates that a level of power density saturation has been attained, beyond which further increases in power density have a marginal effect on the loss in mass of the concrete. In contrast, as the traverse speed is increased then the loss in mass experienced by the concrete decreases, again in a linear manner.

After laser treatment the samples were stored in an uncontrolled environment (open laboratory) and weighed regularly every day for 12 days. The results of these tests are 
illustrated in Fig. 6, which shows clearly that the extent to which the concrete regains mass is a function of the density of the energy deposited on its surface. This is perhaps not surprising since an increase in the energy density increases the likelihood of material ejection or porosity formation. Also, as the energy incident upon the OPC surface of the concrete increases, the HAZ will consequently increase in size.

The general mass regain experienced by the laser treated concrete at the various laser power densities is thought to be the result of the rehydration through contact with the air of the HAZ, which is comprised of unslaked lime (as discussed later). This appears to be a reasonable assumption when one considers that in terms of absolute mass regain, the greatest mass regain occurs with the samples treated with the highest power density. For instance, the concrete sample treated at a power density of $1 \mathrm{~kW} / \mathrm{cm}$ initially reduces in mass to $99.7 \%$ of its original mass, but after 12 days has regained $0.1 \%$ to be finally $99.8 \%$ of its original mass. In contrast, the concrete sample treated at a power density of $3 \mathrm{~kW} / \mathrm{cm}$ initially reduces in mass to $98.4 \%$ of its original mass, but after 12 days has regained some $0.5 \%$ to be finally $98.9 \%$ of its original mass.

\section{THERMAL ANALYSIS}

A number of methods exist that can yield the thermal characteristics of concrete. Arguably the two most effective are Thermogravimetric Analysis (TG) and Differential Thermal Analysis (DTA), which are commonly performed simultaneously. From these techniques it is possible to observe the full range of reactions which occur in the OPC surface of the concrete from ambient temperature up to, and including, the melting temperature. TG-DTA of concrete has been carried out and explained fully by a number of workers ${ }^{11-13}$. Table I details the summary results of these analyses. 


\section{A. The effects of increasing temperature}

\section{Effect on water held in cement paste}

Water is present within concrete in three forms ${ }^{14}$ : (i) Combined Water - water which is combined in the hydrated cement compounds and as such is part of the solid, (ii) Gel Water adsorbed water which is held by surface forces on the gel particles and (iii) Capillary Water free water which is present in the pores beyond the range of the surface forces of the solids in the cement paste. In practice, these divisions are somewhat arbitrary, as low levels of heating can result in the loss of combined water, whilst some gel and capillary water remains. Nonetheless, the divisions serve to outline the general behaviour of the first stages of heating during the HPDL treatment of the OPC surface of the concrete as detailed in Table I- Region I.

The first effect of the heat generated by laser interaction with the OPC surface of the concrete is to weaken the bonds of the bound water layers, causing a reduction in strength. The loss in strength is regained above $120^{\circ} \mathrm{C}$ as the OPC surface of the concrete begins to dry out, resulting in densification and an increase in the van der Wall's forces as the cement gel layers move closer together ${ }^{15}$. This is due to the evaporation of capillary water from large pores near to the surface of the OPC at temperatures between 30 and $120^{\circ} \mathrm{C}^{12}$.

As the temperature increases, so does the rate of evaporation due to the loss of the more tightly bound gel and chemically-bound waters ${ }^{15}$. As the chemically-bound water is released the gel begins to break down, occasioning an increase in porosity and microcracking. Consequently, there is a rapid loss in strength above $300^{\circ} \mathrm{C}^{12}$. The dehydration of ettringite occurs between 100 and $150^{\circ} \mathrm{C}$. With the application of sufficient heat the ettringite may ultimately be converted to $\mathrm{CaO}$ and $\mathrm{Al}_{2} \mathrm{O}_{3}$. Again, this will lead to a loss in strength.

\section{Dehydration of $\mathrm{Ca}(\mathrm{OH})_{2}$}

DTA results show that up to approximately $420^{\circ} \mathrm{C}$ the concrete remains relatively stable ${ }^{11,12}$. Some dehydration occurs, however, and water is also lost from the pores of the cement. But, as one can see from Table I- Region II, this effect is far outweighed by the dehydration of $\mathrm{Ca}(\mathrm{OH})_{2}$ which follows shortly after $420^{\circ} \mathrm{C}$ is exceeded in accordance with 


$$
\mathrm{Ca}(\mathrm{OH})_{2} \rightarrow \mathrm{CaO}+\mathrm{H}_{2} \mathrm{O}
$$

Additionally, the dehydration of the $\mathrm{Ca}(\mathrm{OH})_{2}$ promotes the development of microcracks which begin initially around the $\mathrm{Ca}(\mathrm{OH})_{2}{ }^{12}$. Moreover, this dehydration results in unslaked lime $(\mathrm{CaO})$ which is effectively the $\mathrm{HAZ}$. This $\mathrm{CaO}$ is either located below the glazed surface layer or around the edges of the glaze. Indeed, by using a phenolphthalein indicator followed by water misting, it was possible to clearly discern the HAZ around the laser treated zone on the OPC surface of the concrete, since phenolphthalein is an indicator which is colourless in $\mathrm{CaO}$, turning violet-red in the presence of $\mathrm{Ca}(\mathrm{OH})_{2}$ due to the change in $\mathrm{pH}$.

\section{MECHANICAL, CHEMICAL AND PHYSICAL PROPERTIES}

Current British and international standards in relation to concrete, are concerned only with water sorptivity and compressive strength. Consequently, it was not possible to test the HPDL generated glazes according to, and strictly adhering to, established tests. As such, wherever possible tests based on current standards were developed to investigate specific aspects of particular relevance to the HPDL generated glazes; namely the pull-off strength, the surface roughness, the rupture strength, the wear resistance and the corrosion resistance.

\section{A. Pull-off strength}

In order to ascertain the strength of the bond between the HPDL generated surface glaze on the OPC and the concrete substrate itself, pull-off tests were conducted. For the tests the concrete was prepared as relatively small area samples $(25 \mathrm{~mm} \times 25 \mathrm{~mm})$. High tensile aluminium test dollies were then attached onto the glazed surface and to the axially opposite concrete substrate surface using Araldite epoxy and left to cure for 24 hours. In order to ensure axial accuracy (essential for true results), the test dollies were set in position using identical V-blocks. The samples were placed into an Instron 4507 tensile/compressive test rig by mounting the test dollies into the jaws of the rig. A tensile force was then applied until failure with the energy being simultaneously recorded.

The results obtained varied markedly with changes in the laser operating parameters, as

Fig. 7 shows. A post-test analysis of the samples showed that the material failed 
approximately below the laser treated surface in the HAZ. Within the optimum laser operating parameters the average bond strength of the glaze was recorded as $24 \mathrm{MPa}$. This compares with $63 \mathrm{MPa}$ for the untreated OPC surface of concrete.

\section{B. Rupture strength}

Tests were conducted to determine the rupture strength of the OPC glaze. Test samples were prepared as described above. The samples were placed onto the sample stage of the Instron 4507 tensile/compressive test rig and then subjected to a compressive rupture force until the OPC glaze failed (cracked), with the energy being simultaneously recorded. The rupture force was applied by means of a high tensile steel indentor with a $1 \mathrm{~mm}$ radius point. The results of the tests revealed that the average rupture strength of the OPC glaze was only $0.8 \mathrm{~J}$, whilst the rupture strength of the untreated OPC surface was some $4.3 \mathrm{~J}$.

\section{Water sorptivity testing}

In order to test the water sorption properties of the OPC glaze, or in other words, the effect of the OPC glaze on the water absorption characteristics of the concrete, comparison experiments with the untreated OPC surface were conducted by measuring the water sorptivity. For the experiment the HPDL treated and untreated OPC samples were cut into smaller pieces $(25 \times 25 \mathrm{~mm})$. The tests were conducted in accordance with the standard procedure as used by Hall et al ${ }^{16}$. The samples were dried to a constant weight in an air oven at $65^{\circ} \mathrm{C}$ to ensure all pores were free of water. The OPC laser glaze and the untreated OPC surfaces were then immersed in water and weighed at regular intervals. The side faces of the two samples were shielded from the water by means of an Araldite coating. In order to determine the sorptivity of the OPC glaze and the untreated OPC surface, $i$ was plotted against the square root of time so as to give a straight line, as shown in Fig. 8. $i$ is defined as

$$
i=\frac{\Delta m .1000}{A}
$$

where $\Delta m$ is the cumulative change in mass with time and $A$ is the absorbing surface area. The sorptivity, $S$, the OPC glaze and the untreated OPC surface is simply the gradient of this line. 
As is evident from Fig. 8, the sorptivity of the untreated OPC surface was a typical $0.096 \mathrm{~mm} / \mathrm{min}^{1 / 2}$, compared with $0.043 \mathrm{~mm} / \mathrm{min}^{1 / 2}$ for the laser glazed OPC surface. Thus it is reasonable to conclude that since the laser glazed OPC surface has half the sorption of the untreated OPC surface, then the OPC laser glaze afforded the concrete twice as much resistance to water absorption than the untreated OPC surface. In addition, because the bestfit straight line for the laser glazed OPC surface is below that of the untreated OPC surface, as well as intercepting the axis at a point below the untreated OPC surface, then it can be concluded that rate of absorption is of the laser surface glazed concrete is much less that of the untreated concrete. Furthermore, it is a distinct possibility that the HAZ, which was identified as $\mathrm{CaO}$ resulting from the dehydration of the $\mathrm{Ca}(\mathrm{OH})_{2}$, may, once re-hydrated, act as a barrier towards liquids such as water, therefore augmenting the resistance of the laser glazed OPC surface to water absorption.

\section{Wear life characteristics}

The wear resistance of a material in general is determined primarily by the hardness of the material in comparison with the hardness of other materials with which it comes into contact ${ }^{17}$. However, wear resistance does not always increase with hardness ${ }^{18}$. Tests were therefore conducted in accordance with Lawrence et al ${ }^{19}$ to determine the exact difference in wear resistance characteristics of the OPC glaze and the untreated OPC surface. For experimental purposes the OPC was cut into smaller pieces $(25 \times 25 \mathrm{~mm})$. Half of the samples were then laser treated. All the samples were then weighed and subjected to a friction force for 8 hours, being removed from the machine and weighed at two hourly intervals.

Fig. 9 shows the relationship between weight loss and the friction time for the OPC glaze and the untreated OPC. As one can see, the wear resistance of the OPC glaze is fractionally greater than that of the untreated OPC. However, the OPC glaze shows a significant increase in wear resistance over the untreated OPC surface, with the weight loss being 2 times lower after 4 hours, and 3 times lower after 8 hours.

\section{E. Corrosion resistance}


Concrete surfaces are often subjected to corrosive substances, either as part of the normal service environment and/or as a result of routine cleaning. Therefore corrosion resistance tests based upon BS $6431{ }^{20}$ were conducted using nitric acid, sodium hydroxide and Premier Products MP9 detergent cleaner. The experiments were carried out by dropping small amounts of the corrosive agents in the concentration ratios of $80 \%, 60 \%, 40 \%, 20 \%$ and $10 \%$ on to the surface of the untreated and HPDL glazed OPC surface of concrete at hourly intervals for four hours. The samples were then examined optically and mechanically tested in terms of compressive strength and wear. High concentrations of the various corrosive agents were used principally to accelerate the tests. However, in practice $60 \%$ nitric acid is used within the nuclear processing industry as a solvent for nuclear fuels ${ }^{21}$.

All three substances in the concentrations $80 \%, 60 \%$ and $40 \%$ were seen to immediately attack the untreated OPC surface, with the nitric acid and sodium hydroxide attacking with greater severity than the detergent, whilst the HPDL glazed surface displayed no discernible microstructural changes or signs of devitrification due to corrosion.

Tests conducted according to ASTM C579-91 ${ }^{22}$ revealed that exposure of the untreated OPC surface to the reagents had a significant effect on the compressive strength and the wear resistance of the OPC. As Fig. 10 shows, exposure of the OPC to nitric acid and sodium hydroxide in the concentrations $40-80 \%$ resulted in an average loss of compressive strength of approximately $19-37 \%$. In the case of the detergent a discernible loss in compressive strength only occurred with concentrations above $40 \%$. Here the average loss in compressive strength for concentrations in the range $60-80 \%$ was approximately $17 \%$. This compares with no discernible difference in either the wear resistance or the compressive strength of the HPDL glazed surface.

Fig. 11 shows the variation in wear resistance of the untreated OPC surface when exposed to the reagents with an $80 \%$ concentration. As one can see, the wear resistance is significantly affected, particularly through interaction with the nitric acid and the sodium hydroxide. Here the weight loss was approximately 5 times higher than for the unexposed OPC after 4 hours, and approximately 11 times higher after 8 hours for the nitric acid. In the case of the detergent the weight loss was marginal after both 4 and 8 hours. 


\section{F. Wear life characteristics}

As the results of the mechanical and chemical tests show, the OPC glaze out performed the untreated OPC surface in almost all the test areas. Moreover, the generally superior mechanical and chemical performance of the OPC glaze over untreated OPC suggests that the life characteristics of the OPC glaze may also superior to those of untreated OPC. This was especially true in the case of chemical resistance and water permeability, where the OPC glaze proved to be resistant to both. This marked variation in corrosion resistance and permeability performance is due to the difference in structure of the OPC glaze and the untreated OPC. Whereas the OPC glaze is of an amorphous nature, the untreated OPC is comprised of a porous polycrystalline structure, thus the untreated OPC is readily attacked by acids whilst the amorphous structure of the OPC glaze ensure an increase in acid resistance ${ }^{23}$. However, in any analysis of the wear life of the two materials the in-situ relative thickness of the OPC glaze and the untreated OPC layer on concrete must be considered in order to give a true interpretation of the actual life characteristics, particularly when considering the wear resistance (with and without exposure to corrosive chemical agents). Consequently the increase in wear life can be given by

$$
\begin{aligned}
& \text { Increase in wear life }=\frac{\text { OPC laser glaze wear life }}{\text { Untreated OPC wear life }} \\
& \text { where, } \quad \text { Wear life }=\frac{\text { Density } . \text { Thickness }\left(\mathrm{mg} \cdot \mathrm{cm}^{-3} \cdot \mathrm{cm}\right)}{\text { Wear rate }\left(\mathrm{mg} \cdot \mathrm{cm}^{-2} \cdot \mathrm{h}^{-1}\right)}
\end{aligned}
$$

Table II summarises the wear rate details and the nominal life increase of the OPC glaze over the untreated OPC surface. As Table II shows, the OPC glaze gives an increase in actual life over the untreated OPC surface regardless of the environment. However, as one can see, the increase in actual life of the OPC glaze over the untreated OPC surface varies considerably depending upon the working environment. But, notwithstanding this, arguably the most common working environment for an OPC surface would involve some contact with at least detergent acids, therefore yielding significant economic savings since a HPDL glazed OPC surface lasts around 2.5-times longer than one which is unglazed. 


\section{DISCUSSION}

\section{A. Glaze formation mechanism}

The complex chemistry of the OPC surface of the concrete and the hydration of its various constituents are not yet fully resolved ${ }^{24}$. Notwithstanding this, it is known that the constituents of OPC are minerals which exist as multi-component solid solution chemical compounds. Of particular importance with regards this study, OPC contains in relatively large proportions: $\mathrm{SiO}_{2}(21 \mathrm{wt} \%), \mathrm{Al}_{2} \mathrm{O}_{3}(5 \mathrm{wt} \%)$ and $\mathrm{Fe}_{2} \mathrm{O}_{3}(3 \mathrm{wt} \%)$, which are basic glass network formers and modifiers. Consequently the intense local heating brought about by the incident HPDL beam results in melting of these compounds at around $1283^{\circ} \mathrm{C}$, thereby causing the materials to lose the retained water and form an amorphous glassy material consisting of various calcium-silicate-alumina compounds ${ }^{4}$. Indeed, the amorphous nature of this glaze is verified by the XRD analysis results given in Fig. 12 .

As was mentioned earlier, HPDL interaction with the OPC surface occasioned a dramatic colour change; changing from grey to green. These changes are due to the resultant phase transitions and also, the presence in small concentrations of metal transition ions in various oxidation states within the OPC composition, in particular, ferric ions in the $\mathrm{Fe}^{3+}$ and $\mathrm{Fe}^{2+}$ oxidation state. $\mathrm{Fe}^{3+}$ and $\mathrm{Fe}^{2+}$ ions are known to give rise to green and blue colours respectively when subjected to intense heating ${ }^{25,}{ }^{26}$. However, if both phases are present within the composition, then the colour is determined by the $\mathrm{Fe}^{3+} / \mathrm{Fe}^{2+}$ ion ratio, resulting in dark blue or black colours ${ }^{25,26}$. Since the surface produced after HPDL treatment was green, then it is reasonable to assume that both phases were not present within the OPC.

\section{Cracking and the formation mechanism}

As Fig. 1 and Fig. 2 show, cracking of the HPDL induced glaze occurred to various degrees. The formation of cracks can be attributed mainly to thermal stresses generated during HPDL irradiation. This is due to the fact that OPC has low thermal conductivity, and, as such, during laser heating a large thermal gradient between the melt zone and the substrate exists which results in the generation of thermal stresses. Additionally, despite the fact that the HPDL surface treatment process is effectively localised in nature, the fact remains that a 
certain amount of the heat generated will be conducted to sections of the OPC where the surface is already glazed. This, combined with existence of a relatively cold OPC substrate means that thermal stresses will be generated. During the heating phase the stresses will be compressive and relieved by plastic deformation, thus precluding crack formation. At high temperatures $\left(T \geq T_{m}\right)$ the stresses can also be relieved ${ }^{27-29}$. However, during cooling when the temperature falls below $T_{m}$, then stresses will accumulate. If the fracture strength of the material is exceeded, then cracking within the melted layer will occur. The thermal stress $\sigma$, induced by a thermal gradient can be calculated using the Kingery equation:

$$
\sigma=\frac{E \alpha \Delta T}{1-v}
$$

where $E$ is Young's modulus, $\Delta T$ is the temperature change, $\alpha$ is the coefficient of thermal expansion and $v$ is Poisson's ratio. More succinctly, $\Delta T$ is the difference between the critical temperature (below which stresses can no longer be relieved) and ambient temperature. For $\mathrm{OPC}$ this is the difference between the melting point, $1283^{\circ} \mathrm{C}$ and ambient temperature $20^{\circ} \mathrm{C}$. So, if it is assumed that the glass formed on the surface of the OPC is similar to soda-limesilica glass because the compositions of the two materials are similar, then by using the following values for pyrex: $E=6.42 \times 10^{4} \mathrm{MN} / \mathrm{m}, \alpha=33 \times 10^{-7} \mathrm{~K}^{-1}, \Delta T=1263^{0} \mathrm{C}$ and $v=0.176$, when the OPC surface of the concrete was irradiated by the HPDL beam the thermal stress produced in the resulting glass according to Equation (4) was around $305 \mathrm{MN} / \mathrm{m}$. Since this is well in excess of the fracture strength of the glass, $120 \mathrm{MN} / \mathrm{m}^{30}$, cracking will occur, and can only be avoided by severe distortion or through the reduction of $\Delta T$ by pre-heating.

As one can see from Fig. 13, under the extreme laser processing conditions of relatively high power density $(3.25 \mathrm{~kW} / \mathrm{cm})$ and slow traverse speed $(120 \mathrm{~mm} / \mathrm{min})$ with an Ar shroud gas, microcracking within the OPC glaze occurred both parallel and perpendicular to the surface. Such findings are in accord with the nature of the tensile stresses produced during cooling of the solidifying layer in terms of the glazed layer section thickness. During cooling of a relatively thin glazed layer, the temperature is almost uniform across the section, therefore it only experiences a two-dimensional stress at the surface. However, for relatively thick section glazes, as is the case in question, the temperature gradient across the depth is present 
along with the gradients at the surface. Consequently the three-dimensional nature of the stresses produces microcracks that are both perpendicular and parallel to the surface ${ }^{29,31}$.

\section{Porosities and the formation mechanism}

From Fig. 1 and Fig. 2 it can be seen that porosities were a common feature of the HPDL induced glaze, varying in size from microscopic pits to large craters depending upon the laser operating parameters. For all instances of porosity formation the mechanism behind their development is the consequence of gas escaping from within the melt and disrupting the surface ${ }^{32}$. With regard to the OPC glaze, the gas is likely to be $\mathrm{CO}_{2}{ }^{9}$. If the laser energy density incident on the $\mathrm{OPC}$ is too low, then the generated $\mathrm{CO}_{2}$ can not escape from the molten OPC surface easily because of the high viscosity of the melt. As such, when the $\mathrm{CO}_{2}$ eventually does penetrate the melt surface, the resultant porosity is not filled by the flow of the melt; since the insufficient energy density is unable to maintain a high enough temperature for an adequate length of time and thus decrease the overall viscosity of the melt ${ }^{31}$. In this case the porosities formed are typically small and shallow, being regular in both periodicity and intensity (Fig. 14 (a)). On the other hand, if the laser energy density incident on the OPC surface of the concrete is too high, then boiling of the surface may happen. At the same time an increase in $\mathrm{CO}_{2}$ formation may occur within the melt. These individual pockets of $\mathrm{CO}_{2}$ formation may combine and rise to surface of the melt. Once the energy density decreases (as the laser traverses away), then the additional $\mathrm{CO}_{2}$ will attempt to escape from the molten surface. However, as Fig. 14 shows schematically, the solidifying melt will prevent this, causing bubbles to form (a). The excessive $\mathrm{CO}_{2}$ gas pressure will firstly cause the bubbles to expand (b) and ultimately rupture the walls of the bubbles (c), creating a sharp 'knife edge' porosity ${ }^{3,9}$. These types of porosity are usually large, deep and randomly spaced.

\section{Shroud gas effects}

Because of its open structure, it is possible for gases to dissolve molecularly in glass, and, if the gas molecule is small enough, it can diffuse rapidly in a simple glass such as fused 
silica ${ }^{30}$. Indeed, gases such as $\mathrm{H}_{2}$ and $\mathrm{O}_{2}$ are known to dissolve molecularly in glass and can also react with the glass network ${ }^{30}$. The quality of the glazes produced, in terms of porosity and microcracks, was greatly influenced by the type of shroud gas employed. When using both $\mathrm{Ar}$ and $\mathrm{O}_{2}$ shroud gases the surfaces appeared undulated and displayed porosities. However, the crack density in the Ar shroud gas sample was found to be higher than that of the sample treated with an $\mathrm{O}_{2}$ shield gas.

The use of $\mathrm{O}_{2}$ as the shroud gas significantly reduced the number of microcracks and porosities within the OPC glaze. This indicates that the $\mathrm{O}_{2}$ interacted with the glass network increasing the heat generation and subsequently the fluidity of the melt. In contrast, the Ar did not interact with the glass network and was consequently trapped within the more viscous melt in the form of bubbles. As such, when employing $\mathrm{O}_{2}$, inherent gas bubbles generated during vitrification of the OPC escaped from the melt more readily due to its lower viscosity, thus reducing the number of porosities. In addition, as the cooling curves for the HPDL treated OPC surface of the concrete shown in Fig. 15 indicate, when the laser beam had been removed, the cooling of the OPC was faster when an Ar shroud gas as opposed to an $\mathrm{O}_{2}$ shroud gas was employed. Therefore, the length of time that the OPC surface of the concrete is of a sufficient fluidity to allow the generated gas bubbles to escape the surface easily is much reduced, resulting in porosities and microcracks.

\section{B. Stability to devitrification}

Despite the fact that HPDL interaction with the OPC surface of the concrete generates an amorphous glaze, it is possible for the OPC glaze to become crystalline through the destruction of the glassy state by means of a process known as devitrification. This can occur as either the breakdown of the glass surface by corrosion or weathering, or as a result of the overall composition remaining unchanged while the crystals separate in the glassy medium, therefore destroying the glassy state. This process being entirely dependant upon the temperature and composition of the glass.

A high magnification SEM examination of the OPC glaze revealed no evidence of devitrification was observed within the OPC glaze. This indicates that exposure of the OPC 
glaze to the extremely harsh reagents detailed previously did not cause the glaze to devitrify. Nor did the glass devitrify as a result of favourable high temperatures and glass composition. This is of great significance since devitrification in this manner is the result of the movement of atoms to allow orientation and the presence of crystallisation centres. Such centres occur usually at the glass/air boundary, around a porosity ${ }^{17}$. Clearly, as Fig. 1 and Fig. 2 show, porosities were in general a common feature of the OPC glaze. Furthermore, it is highly likely that the composition of the OPC glaze also played an important part in the stability to devitrification of the glaze. In particular it is known that compounds such as $\mathrm{Al}_{2} \mathrm{O}_{3}$ and $\mathrm{MgO}$ (which an EDX analysis showed were present in abundance in the OPC glaze) are known to be very useful in assuaging devitrification problems ${ }^{17}$. This is because the inclusion of such compounds within the OPC glaze composition creates an glaze without a high liquidus temperature and therefore a reduced tendency towards devitrification ${ }^{17}$.

\section{CONCLUSIONS}

Using a $60 \mathrm{~W}-\mathrm{cw}$ high power diode laser (HPDL), glazing of the ordinary Portland cement (OPC) surface of concrete was successfully demonstrated with power densities as low as $750 \mathrm{~W} / \mathrm{cm}$ and at rates up to $480 \mathrm{~mm} / \mathrm{min}$.

Thermal analysis techniques were used to identify the degradation reactions and the temperatures at which they occur. In particular, the dehydration of $\mathrm{Ca}(\mathrm{OH})_{2}$ in the cement matrix was ascertained as forming the heat affected zone (HAZ). Cracks and porosities were common features of the laser glaze. Cracking was identified as being due to the generation of thermal stresses in excess of the fracture strength of the glaze. Porosities are believed to be the result of $\mathrm{CO}_{2}$ formation which ruptures the molten surface as it forms bubbles in an attempt to escape into the atmosphere.

The use of an $\mathrm{O}_{2}$ shroud gas, as opposed to Ar, significantly reduced the number of microcracks and porosities within the OPC glaze. This is believed to be due to $\mathrm{O}_{2}$ interacting with the glass network and thus increasing the heat generation and subsequently the fluidity of the melt. In contrast, the Ar did not interact with the glass network and was consequently trapped within the more viscous melt in the form of bubbles. As such, when employing $\mathrm{O}_{2}$, 
inherent gas bubbles generated during the laser vitrification of the OPC escaped from the melt more readily due to its lower viscosity, thus reducing the number of porosities and microcracks.

Life assessment testing revealed that the OPC glaze had an increase in wear life of 1.3 to 14.8 times over an untreated OPC surface, depending upon the corrosive environment. Mechanical testing of the seals revealed that the average rupture strength of the OPC glaze was only $0.8 \mathrm{~J}$, whilst the rupture strength of the untreated OPC surface was some $4.3 \mathrm{~J}$. The average bond strength of the glaze was recorded as $24 \mathrm{MPa}$. This compares with $63 \mathrm{MPa}$ for the untreated surface of the OPC. Clearly, the economic and material benefits to be gained from the deployment of such an effective and efficient coating on OPC could be significant. 


\section{References}

1. K. Sugita, M. Mori, T. Fujioka, Concrete Eng. 24, 13 (1986).

2. M. Hamasaki, in Proceedings of The International Symposium on Laser Processing (SPIE, Bellingham, 1987), pp 158-167.

3. H. Yoshizawa, S. Wignarajah, H. Saito, Trans. Japan Welding Soc. 20, 31-36 (1989).

4. L. Li, P.J. Modern, W.M. Steen, "Laser Fixing and Sealing of Radioactive Contamination on Concrete Surfaces" Proceedings of LAMP '92, June 1992 (High Temperature Society of Japan, Osaka), pp 843-848.

5. L. Li, W.M. Steen, P.J. Modern, in Proceedings of ISLOE '93, (National University of Singapore, Singapore, 1994), pp 25-30.

6. L. Li, W.M. Steen, P.J. Modern, J.T. Spencer, in Proceedings of RECOD '94 (SPIE, Bellingham, 1994), pp 24-28.

7. L. Li, W.M. Steen, P.J. Modern, J.T. Spencer, Proceedings of EUROPTO '94: Laser Materials Processing and Machining (SPIE, Bellingham, WA, 1994), pp 84-95.

8. K. Sugimoto, S. Wignarajah, K. Nagasi, S. Yasu, "Fundamental Study on Laser treatment of Architectural Materials" Proceedings of ICALEO '90, November 1990 (Laser Institute of America, Orlando, FL), pp 302-312.

9. S. Wignarajah, K. Sugimoto, K. Nagai, "Effect of Laser Surface Treatment on the Physical Characteristics and Mechanical Behaviour of Cement Base Materials" Proceedings of ICALEO '92, October 1992 (Laser Institute of America, Orlando, FL), pp 383-393.

10. T.I. Borodina, G.E. Valyano, N.I. Ibragimov, E.P. Pakhomov, A.I. Romanov, L.G. Smirnova, P.K. Khabibulaev, J. Phys. and Chem. of Mater. Treatment, 25, 541-546 (1995).

11. A. Petzold, M. Rohrs, Concrete for High Temperatures (MacLaren \& Sons, London, 1970), pp 40-85.

12. U. Schneider, U. Diederichs, Betonwerk \& Fertigteil - Technik, 3, 141-149 (1981).

13. K.J. Blair: PhD Thesis, University of Liverpool (1996). 
14. I. Soroka, Portland Cement Paste and Concrete (Macmillan Press, London, 1979), pp 147-166.

15. W.P.S. Dias, G.A. Khoury, P.J.E. Sullivan, ACI Mater. J. 87, 160-166 (1990).

16. C. Hall, K.M. Tse, Building and Environment, 21, 113-118 (1986).

17. J.H. Dickson, Glass: A Handbook for Students and Technicians (Hutchinsons Scientific and Technical Publications, London, 1951), pp 108-148.

18. A. Petitbon, L. Boquet, D. Delsart, Surf. \& Coat. Tech. 49, 57-61 (1991).

19. J. Lawrence, L. Li, J.T. Spencer, Optics \& Laser Tech. 30, 215-223 (1998).

20. BS 6431: Part 19: 1984, Ceramic Wall and Floor Tiles: Method for Determination of Chemical Resistance. Glazed Tiles.

21. J.T. Spencer, Letter to Author, October 1997.

22. ASTM C597-91, Standards Test Method for Compressive Strength of Chemical-Resistant Mortars, Grouts, Monolithic Surfacings and Polymer Concretes.

23. V.V. Vargin, Technology of Enamels (MacLaren \& Sons, London, 1965), pp 125-128.

24. W. Czernin, Cement Chemistry and Physics for Civil Engineers (Bauverlag, Berlin, 1980), pp 124-131.

25. G.D. Taylor, Construction Materials (Longman Scientific \& Technical, London, 1991), pp 31-34.

26. N. Jackson, R.K. Dhir, Civil Engineering Materials, (MacMillan Press, New York, 1992), pp 90-92.

27. F.S. Galasso, R. Veltri, Ceramics Bull. 62, 253-255 (1983).

28. R. Sivakumer, B.L. Mordike, J. Surf. Eng. 4, 127-140 (1988).

29. J. Mazumder, Optical Eng. 30, 1208-1219 (1991).

30. R.H. Doremus, Glass Science (John Wiley \& Sons, New York, 1994), pp 154-160.

31. Z. Lui, PhD Thesis, University of Liverpool (1991).

32. M.H. Lewis, Glasses and Glass-Ceramics (Chapman \& Hall, London, 1989), pp 88-95. 


\section{List of Figures}

FIG. 1. Relationship between OPC surface of concrete laser melt depth and laser power density.

FIG. 2. Effects of laser interaction on OPC surface of concrete at various laser power densities. (a) $0.4 \mathrm{~kW} \mathrm{~cm}^{-2}$, (b) $2 \mathrm{~kW} \mathrm{~cm}^{-2}$ and (c) $3 \mathrm{~kW} \mathrm{~cm}{ }^{-2} .240 \mathrm{~mm} \mathrm{~min}^{-1}$

FIG. 3. Relationship between OPC surface of concrete laser melt depth and traverse speed.

FIG. 4. Effects of laser interaction on OPC surface of concrete at various traverse speeds.

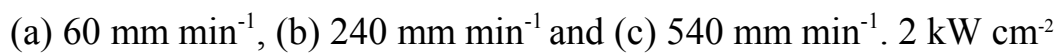

FIG. 5. Relationship between concrete percentage of original mass loss and laser power density and traverse speed.

FIG. 6. Mass regained by concrete after laser treatment with time.

(240 $\mathrm{mm} \mathrm{min}^{-1}$ traverse speed)

FIG. 7. Relationship between pull-off strength of laser glaze with laser operating parameters.

FIG. 8. Water absorption for the untreated and laser glazed OPC surfaces.

FIG. 9. Relationship between weight loss and friction time for the laser generated glaze and the untreated OPC.

FIG. 10. Variation in compressive strength of the untreated OPC with reagent type and reagent concentration.

FIG. 11.Relationship between weight loss and friction time for the untreated OPC with different reagent types at the maximum concentration (80\%).

FIG. 12. XRD analysis of the OPC laser glaze.

FIG. 13. SEM cross-section view of the OPC laser glaze showing microcracks parallel and perpendicular to the surface.

FIG. 14. Illustration of the large 'knife edge' porosity formation.

FIG. 15. Best-fit cooling curves for the laser glazed OPC surface of concrete when $\mathrm{Ar}$ and $\mathrm{O}_{2}$ shroud gases are employed. 
Fig. 1

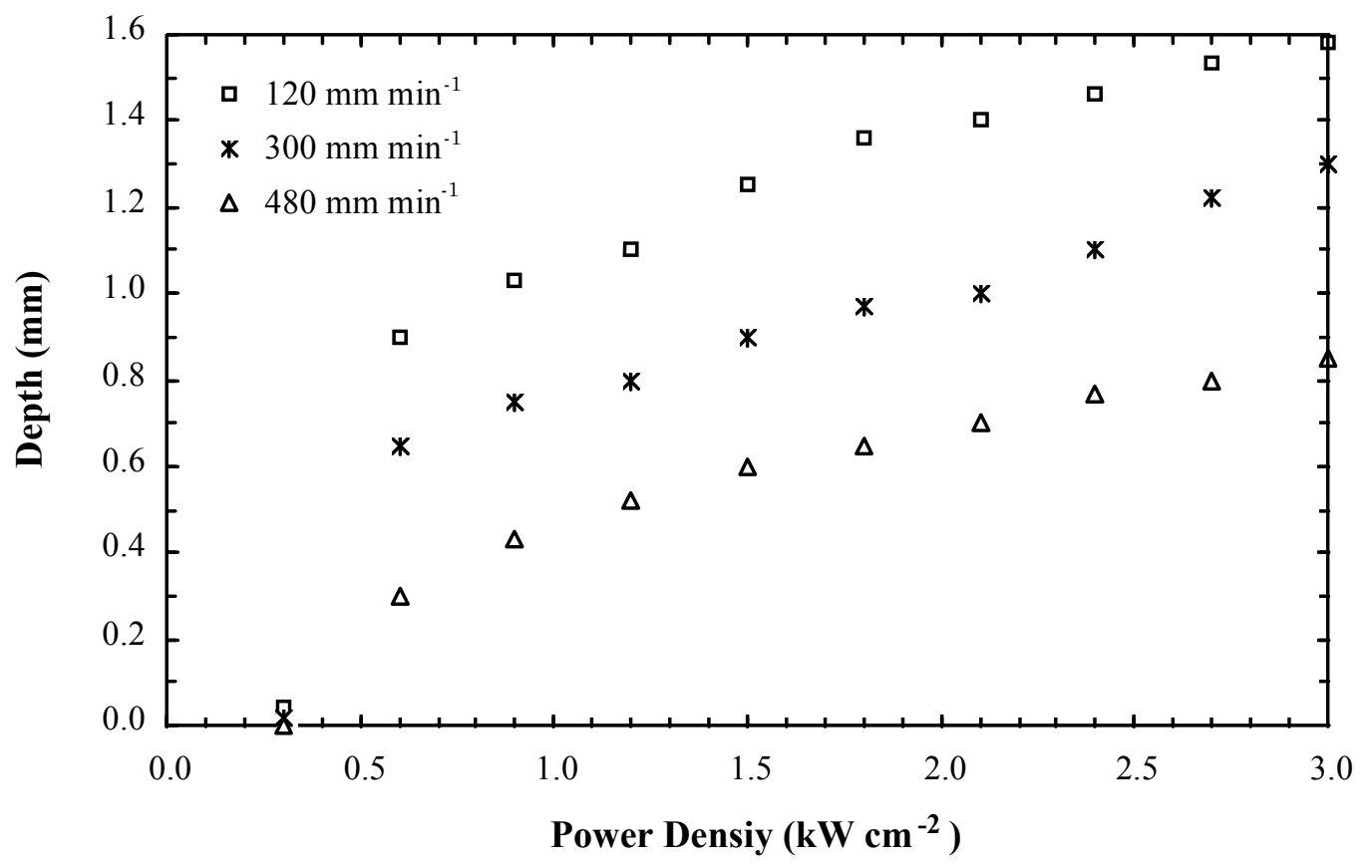


Fig. 2

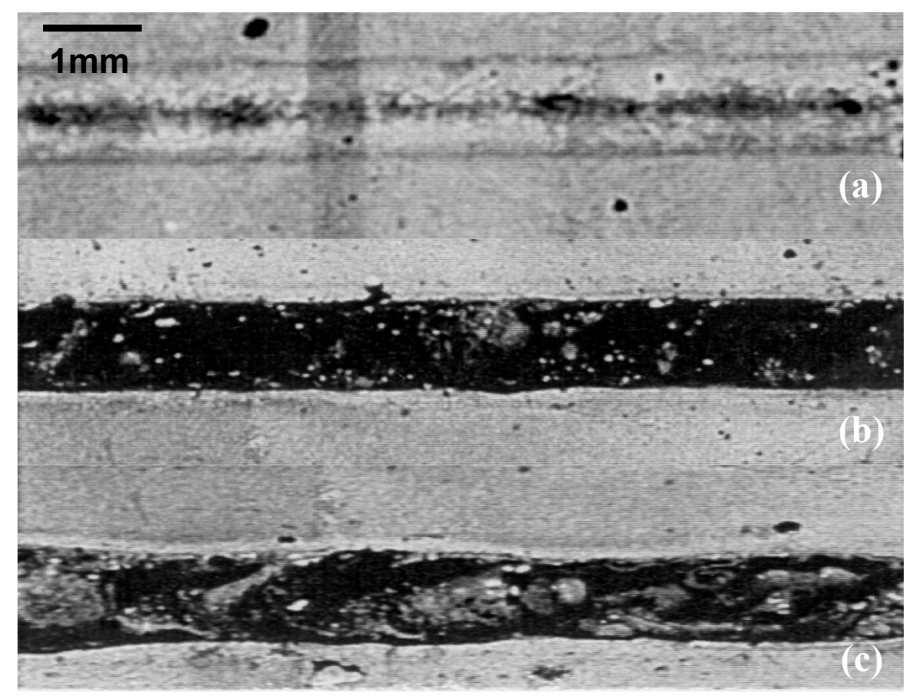


Fig. 3

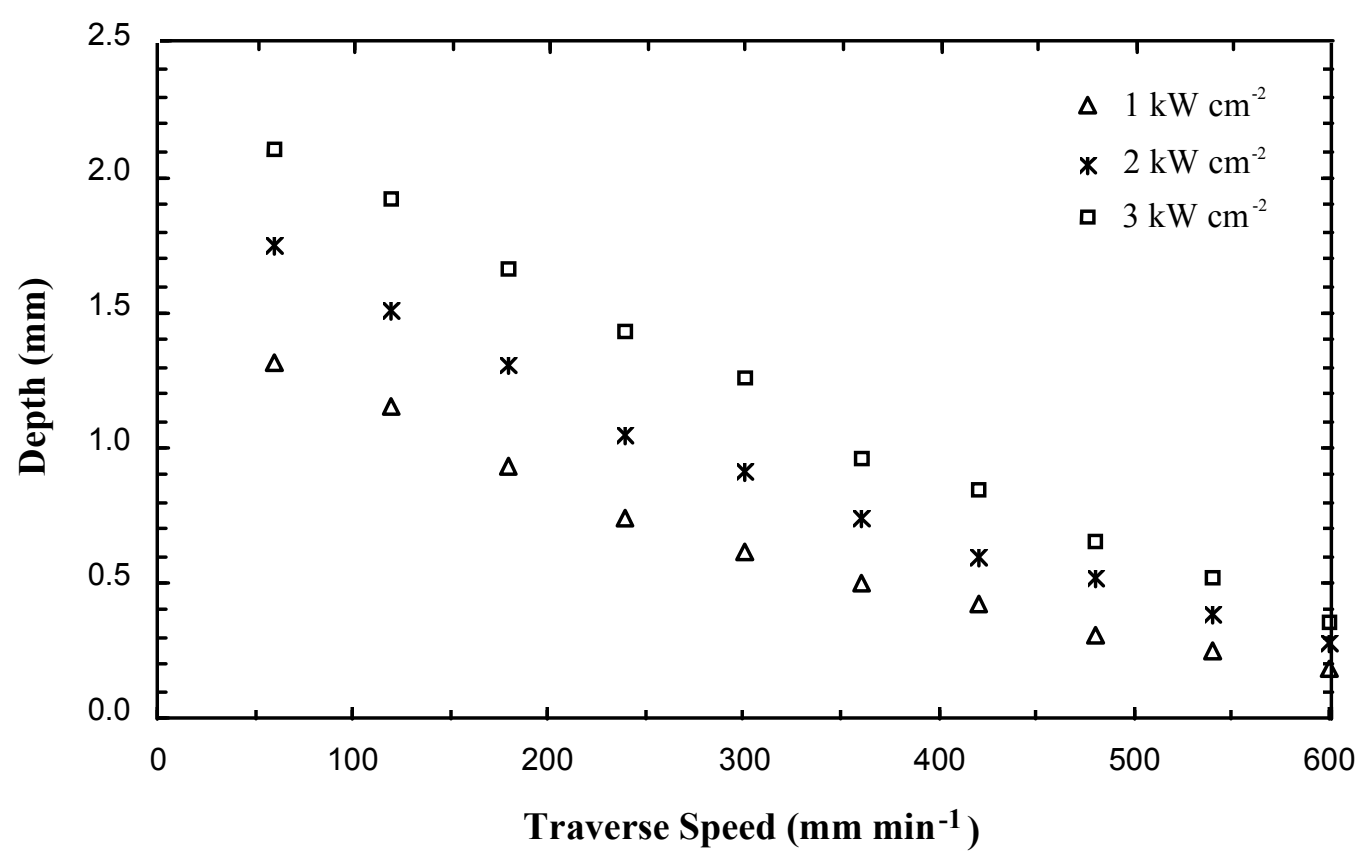


Fig. 4

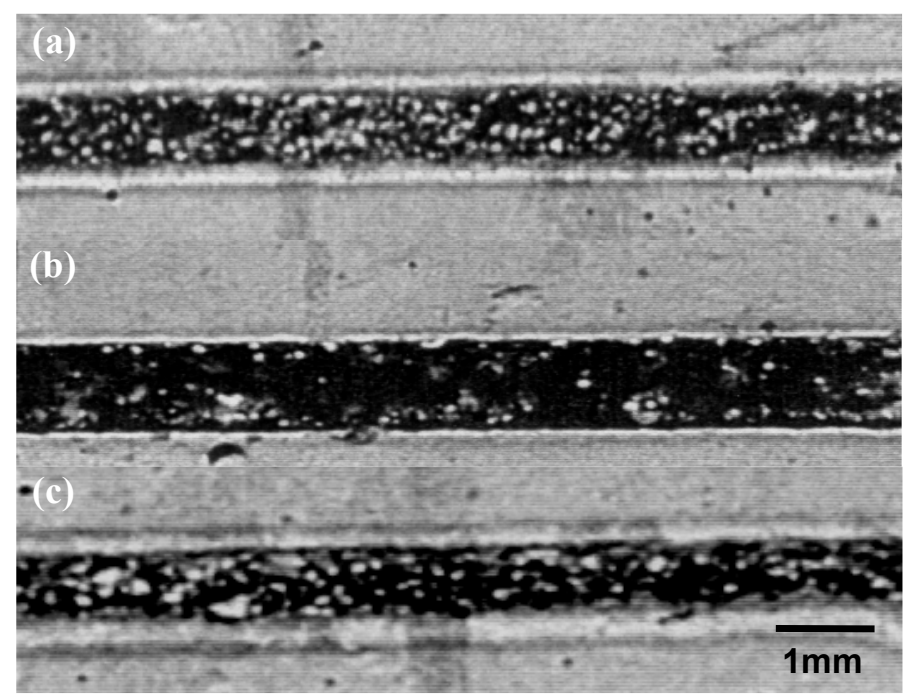


Fig. 5

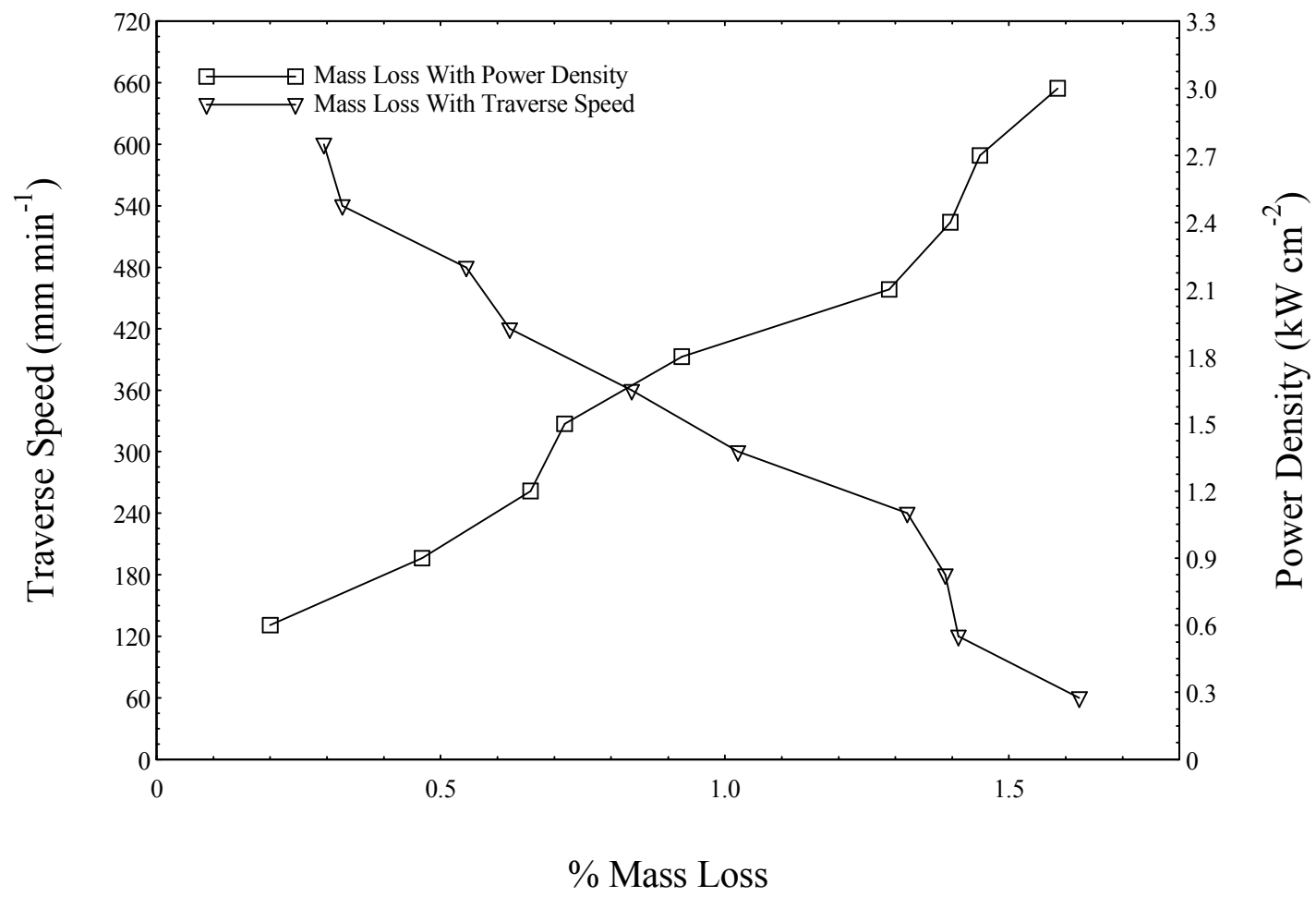


Fig. 6

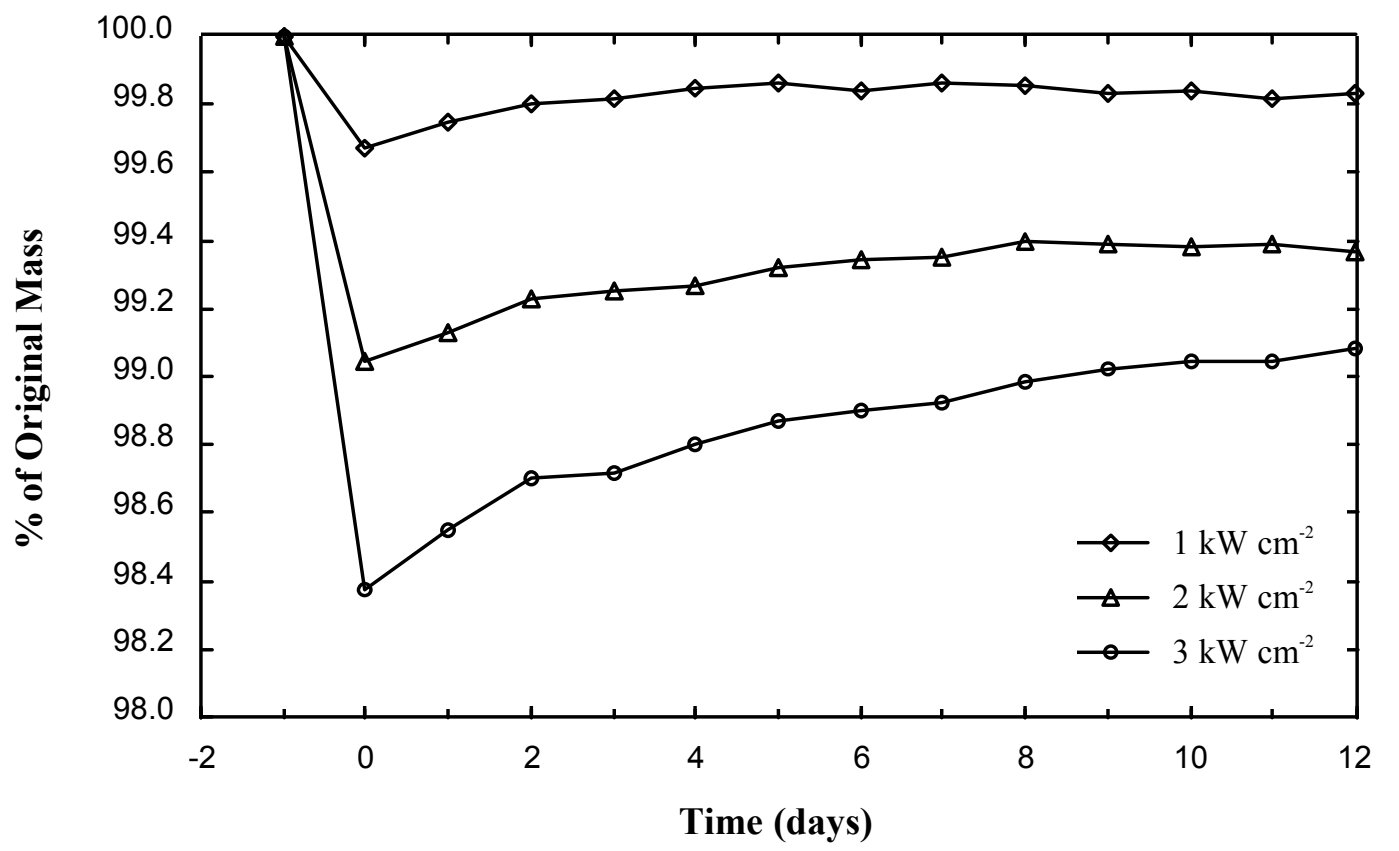


Fig. 7

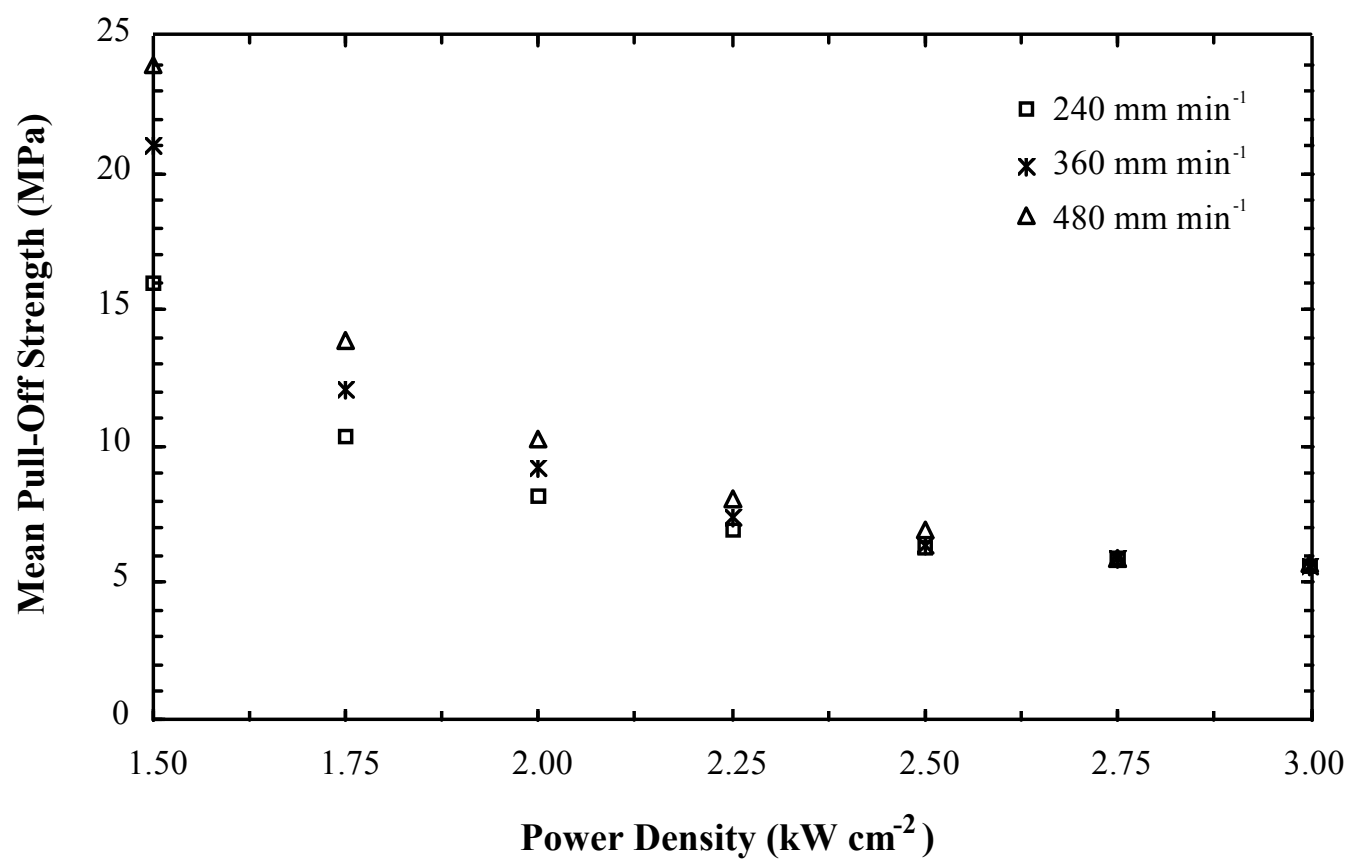


Fig. 8

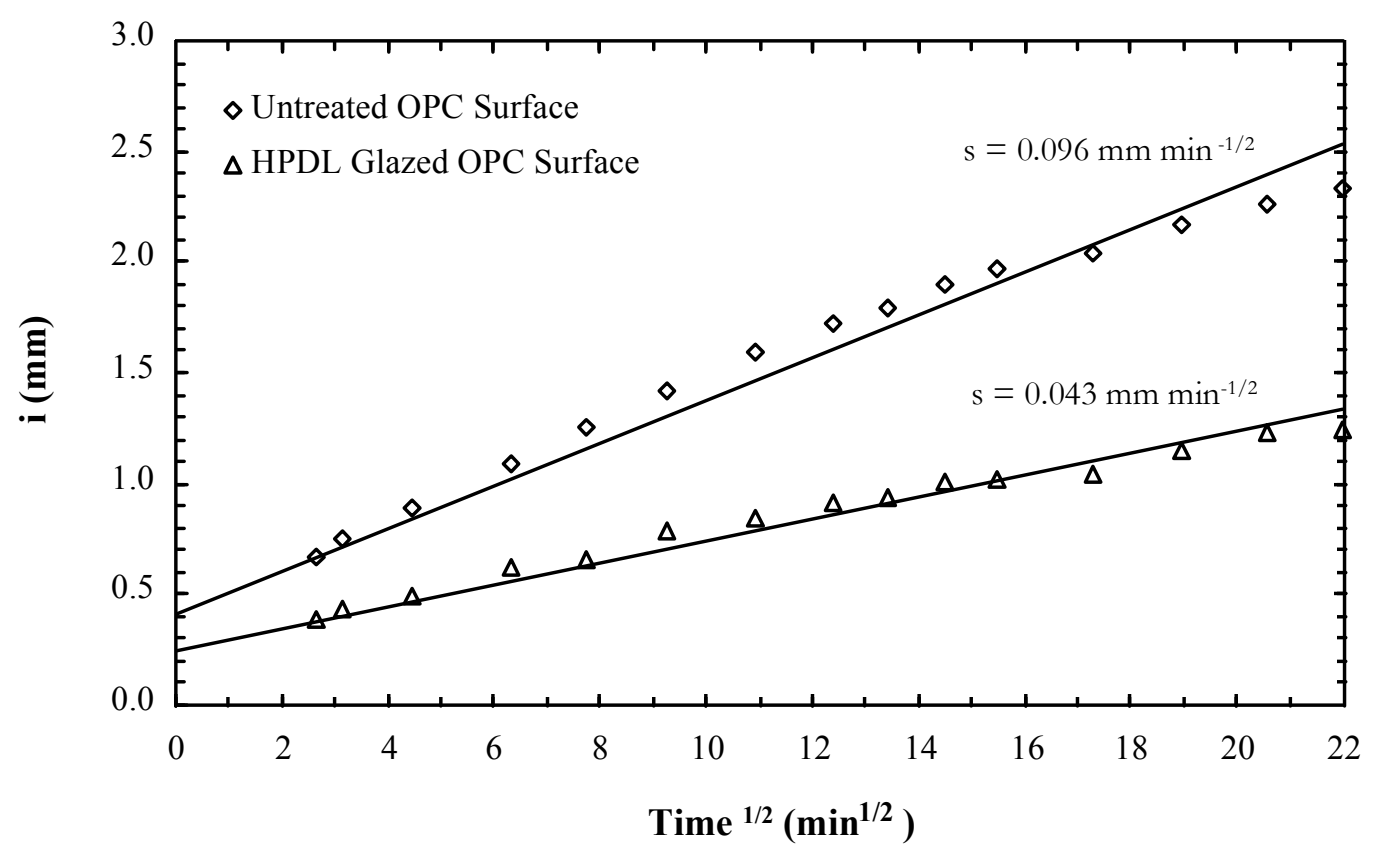


Fig. 9

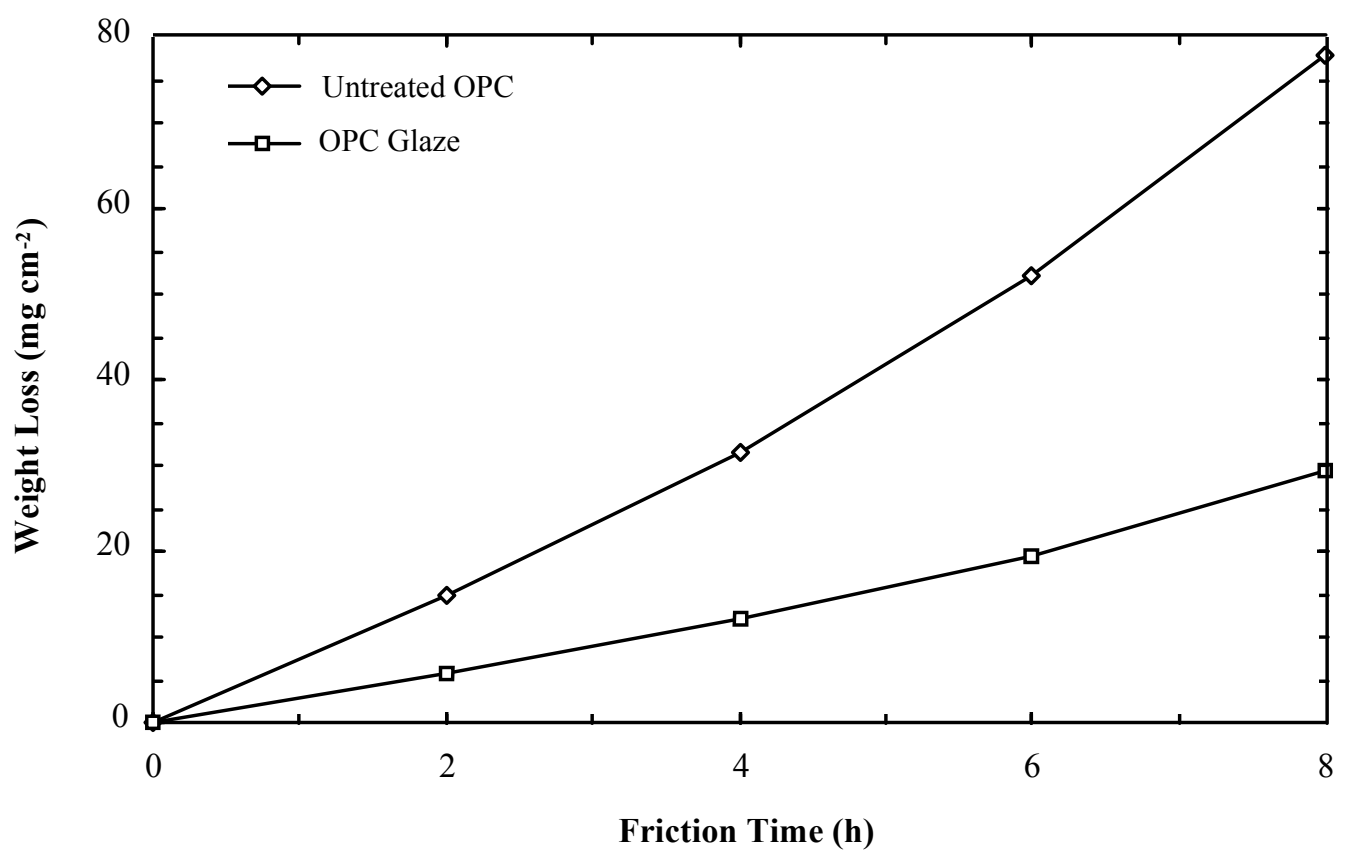


Fig. 10

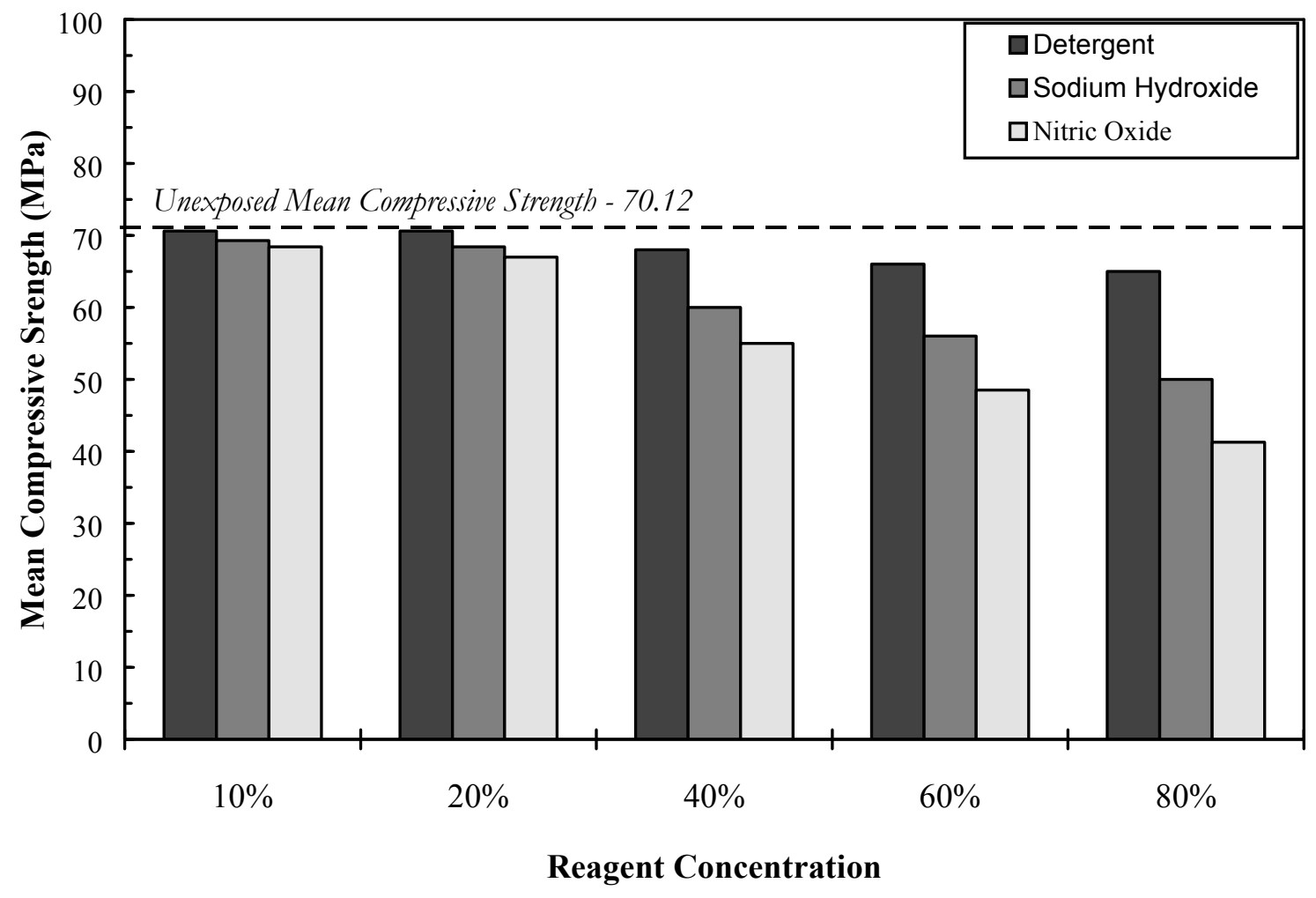


Fig. 11

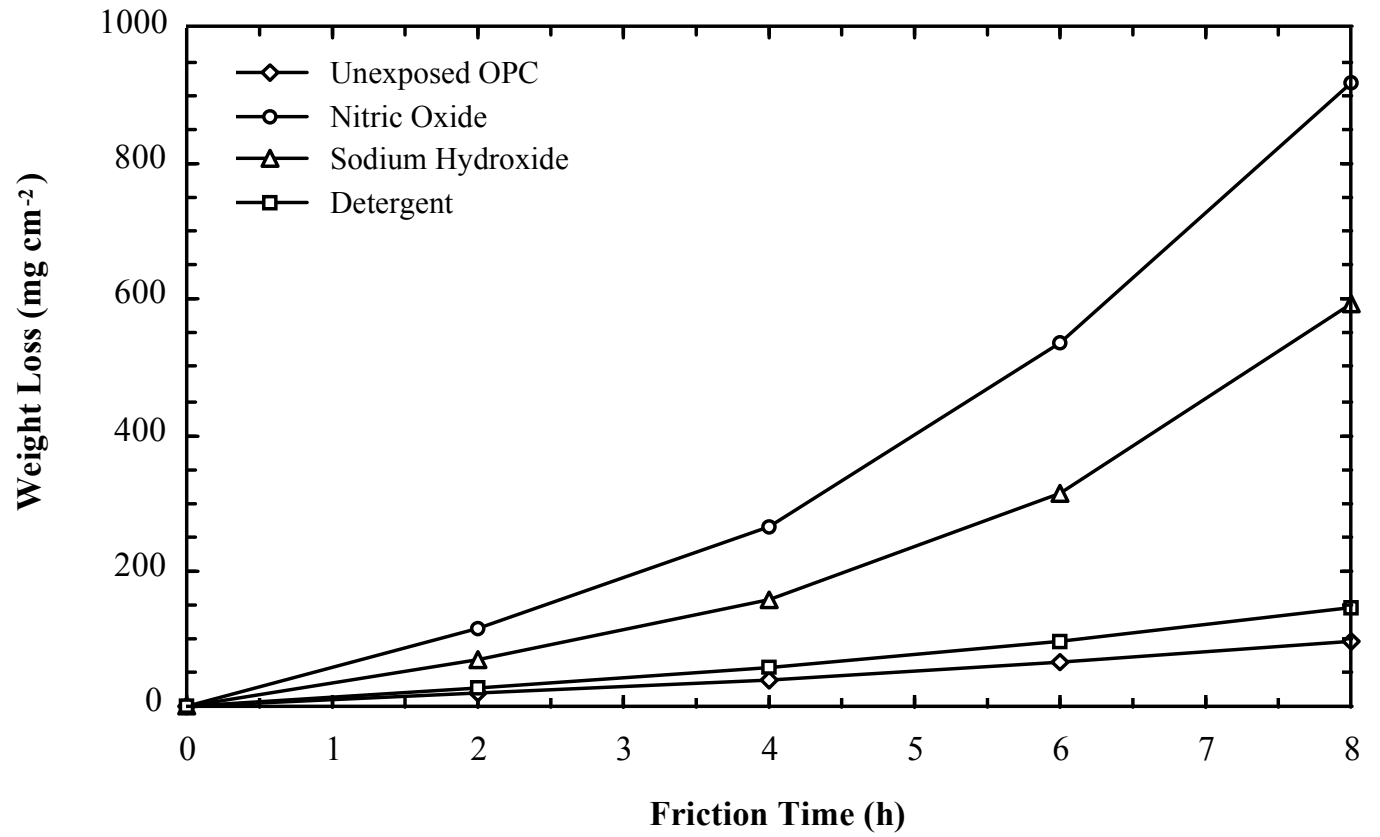


Fig. 12

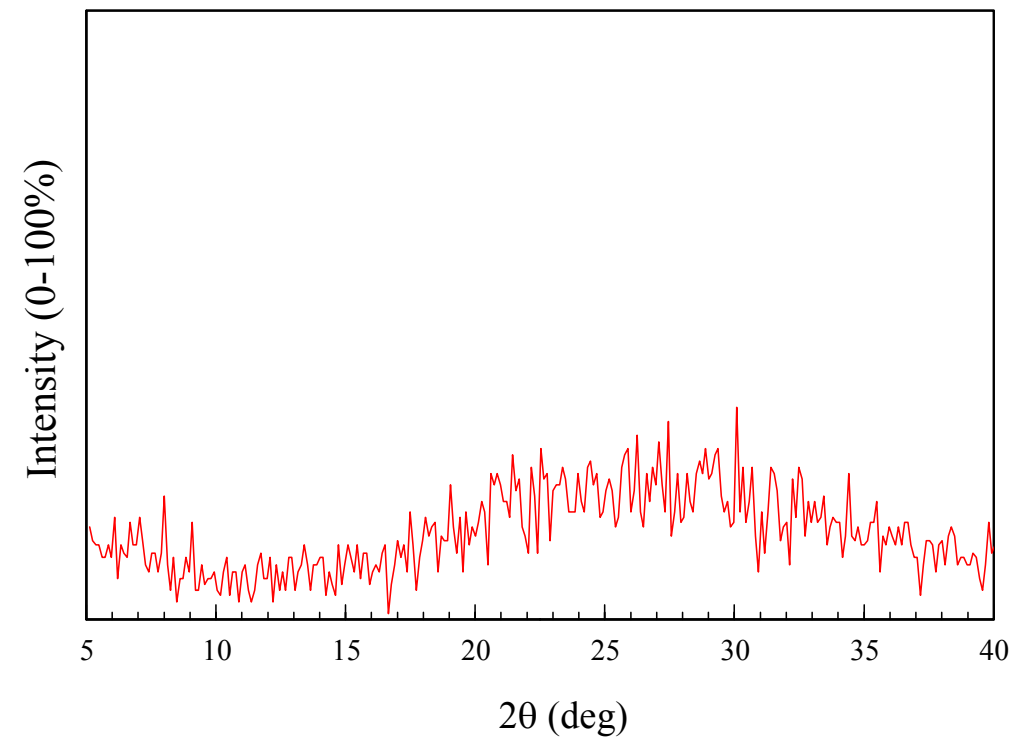


Fig. 13

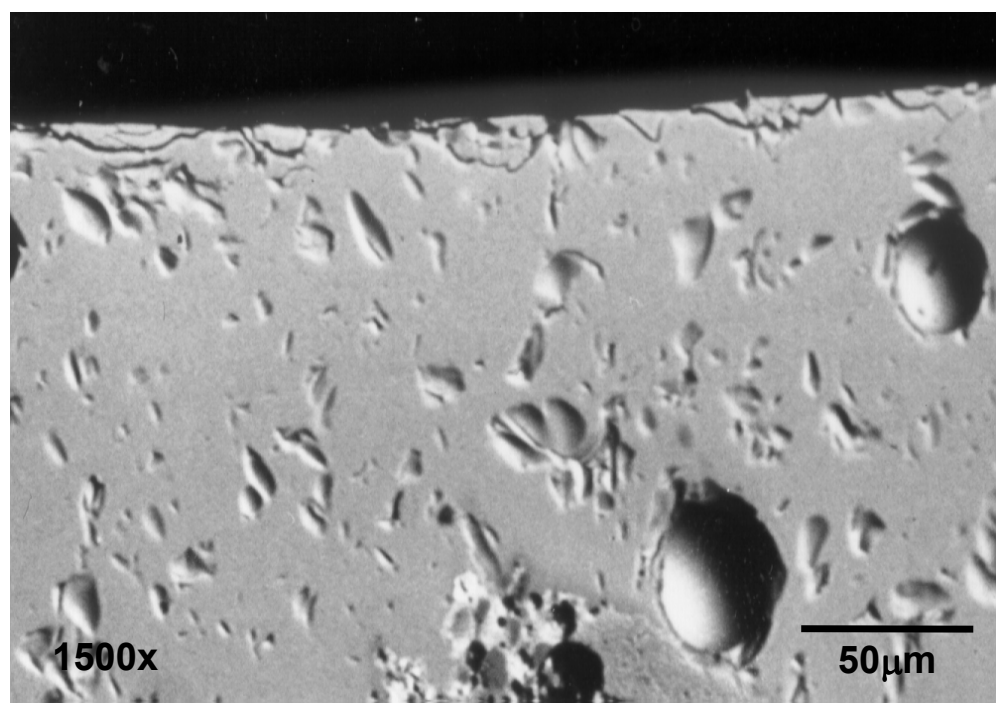


Fig. 14

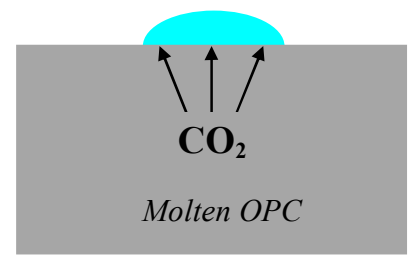

(a)

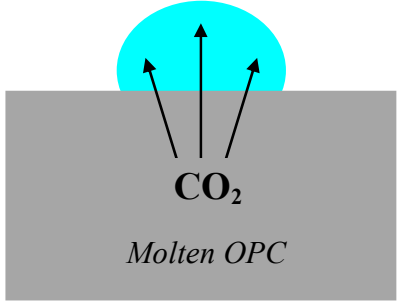

(b)

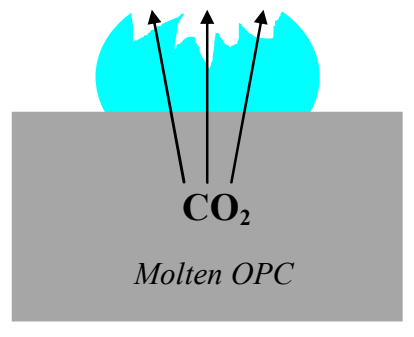

(c) 
Fig. 15

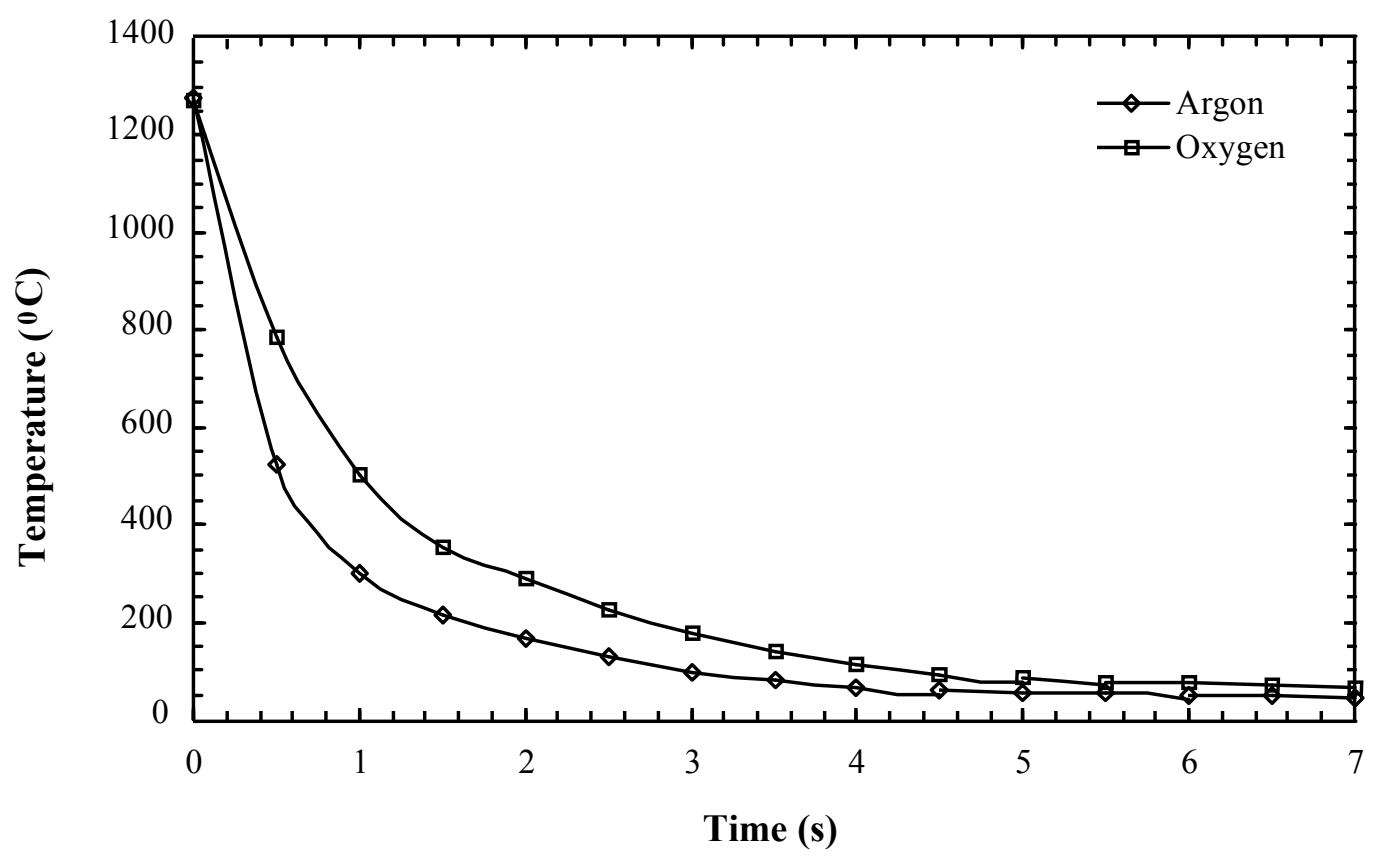




\section{List of tables}

Table 1. Summary details of previously conducted TG-DTA findings [11-13].

Table II. Wear rate details and the nominal life increase of the OPC laser glaze over untreated $\mathrm{OPC}$ in various corrosive environments. 
Table I.

\begin{tabular}{|c|c|c|c|}
\hline Region & Temperature & Observation & Event \\
\hline $\mathrm{I}$ & & & Loss in mass of $4.84 \%$. \\
\hline$\left(20-420^{\circ} \mathrm{C}\right)$ & $\begin{array}{l}114^{0} \mathrm{C} \\
156^{0} \mathrm{C}\end{array}$ & Double Endothermic Peak & $\begin{array}{l}\text { Water loss from hydrates ettringite } \\
\text { and ferrite. }\end{array}$ \\
\hline $\begin{array}{c}\mathrm{II} \\
\left(421-517^{0} \mathrm{C}\right)\end{array}$ & $462^{\circ} \mathrm{C}$ & Sharp Endothermic Peak & $\begin{array}{l}\text { Loss in mass of } 1.01 \% \text {. } \\
\text { Water loss from } \mathrm{Ca}(\mathrm{OH})_{2} \text { resulting } \\
\text { in the formation } \mathrm{CaO} .\end{array}$ \\
\hline \multirow[t]{2}{*}{$\begin{array}{c}\text { III } \\
\left(518-875^{\circ} \mathrm{C}\right)\end{array}$} & $578^{\circ} \mathrm{C}$ & Sharp Endothermic Peak & $\begin{array}{l}\text { Loss in mass of } 13.45 \% \text {. } \\
\alpha \rightarrow \beta \text { solid state quartz phase } \\
\text { transition. }\end{array}$ \\
\hline & $812^{0} \mathrm{C}$ & Large Endothermic Peak & $\begin{array}{l}\text { Loss of } \mathrm{CO}_{2} \text { form } \mathrm{CaCO}_{3} \text { resulting } \\
\text { in the formation of } \mathrm{CaO} \text {. }\end{array}$ \\
\hline $\begin{array}{c}\text { IV } \\
\left(876-1283^{0} \mathrm{C}\right)\end{array}$ & $417^{\circ} \mathrm{C}$ & Endothermic Peak Cluster & $\begin{array}{l}\text { Loss in mass of } 1.49 \% . \\
\text { Fusion (vitrification) of OPC. }\end{array}$ \\
\hline
\end{tabular}


Table II.

\begin{tabular}{ccccccc}
\hline & & & \multicolumn{3}{c}{ Wear Rate $\left(\mathbf{m g . c m}^{-\mathbf{2}} \mathbf{h}^{\mathbf{- 1}}\right)$} \\
& Density & Thickness & Unexposed & Detergent & $\mathbf{N a O H}$ & HNO $_{\mathbf{3}}$ \\
\hline Untreated OPC & $2220\left(\mathrm{~kg} \mathrm{~m}^{-3}\right)$ & $1500(\mu \mathrm{m})$ & 9.8 & 18.5 & 73.8 & 114.8 \\
OPC Laser Glaze & $2000\left(\mathrm{~kg} \mathrm{~m}^{-3}\right)$ & $750(\mu \mathrm{m})$ & 3.5 & 3.5 & 3.5 & 3.5 \\
\hline Increase in Wear Life & $\sim$ & $\sim$ & $\mathbf{1 . 3}$ & $\mathbf{2 . 4}$ & $\mathbf{9 . 5}$ & $\mathbf{1 4 . 8}$ \\
\hline
\end{tabular}

